\title{
Pathophysiology of Brain Ischemia: Penumbra, Gene Expression, and Future Therapeutic Options
}

\author{
B.J. Schaller ${ }^{a}$ M. Bähr ${ }^{b}$ M. Buchfelder ${ }^{a}$ \\ ${ }^{a}$ Department of Neurosurgery and b ${ }^{b}$ Department of Neurology, University of Göttingen, Göttingen, Germany
}

The ischemic penumbra represents a specific tissue region surrounding the ischemic core that has been identified from more than $48 \mathrm{~h}$ after stroke in patients, and that has intermediate perfusion, where cells depolarize intermittently [1]. Without treatment, the penumbra often progresses to infarction owing to the effects of ongoing excitotoxicity, spreading depolarization and postischemic inflammation. Maintenance of perfusion pressure in this region and hence survival of neurons within this electrophysiologically dynamic area of tissue is critical for the minimization of long-term damage. Because of these factors, the penumbra clearly has a limited life span and appears to undergo irreversible damage within a few hours unless reperfusion is initiated and/or neuroprotective therapy administered.

Sharp et al. [2] proposed the existence of multiple 'molecular penumbras' that represent zones within which differential patterns of gene expression are determined on the basis of differential perfusion levels. A number of studies have examined gene regulation after ischemic stroke in animal models and in humans, using cDNAprinted microarrays [3-5], and have demonstrated deregulation of numerous novel genes. Some ischemia-hypoxia response genes were upregulated and some were downregulated; these consisted of immediate early genes, heat shock proteins, antioxidative enzymes, trophic fac- tors, and genes involved in RNA metabolism, inflammation, and cell signaling [6]. All these studies suggest that there is a need to determine the time course of expression of neuroregulatory, angiogenic and apoptotic factors and their associated enzymes after ischemic stroke in humans underscoring the enormous complexity of ischemic biology and direct attention to potentially novel mechanisms for future exploration [6]. Such detailed analyses may allow for the design of new 'neuroprotective' treatment strategies, based on the observed signaling cascades in affected neurons in the ischemic territory [7].

The study by Vikman and Edvinsson [5] substantially supports the interesting hypothesis of upregulation of $G$ protein-coupled receptors to be triggered in part by changes in shear stress as an important mechanism in the process following human stroke hypothesizing that cerebral ischemia induces enhanced transcription of some $G$ protein-coupled receptors specifically in the artery leading to the ischemic region. The upregulation of these receptors and activation of previously mentioned processes together could help to explain the disruption in blood flow seen after cerebral ischemia (resulting locally in both enhanced vasoconstriction and in hypertrophy of the smooth muscle cells), possibly involved in the penumbral zone expansion. When rigorously tested and independently validated, data from large-scale gene expression analyses

\section{KARGER \\ Fax +4161306 1234 E-Mail karger@karger.ch} www.karger.com
(C) 2005 S. Karger AG, Basel 0014-3022/05/0544-0179\$22.00/0

Accessible online at:

www.karger.com/ene
B.J. Schaller, MD

Universitätsspital

CH-8000 Zürich (Switzerland) 
provide new insights into the aggregate genetic control of stroke injury, and the interrelationship of important cellular events within the damaged region.

The molecular signals for poststroke angiogenesis begin within hours of initial cerebral ischemia, with sequential increases in message for initially destabilizing combinations of vascular growth factors and growth factor receptors, followed by growth factor combinations that promote endothelial cell division and stabilization. The overlap in molecular signaling between poststroke angiogenesis, neurogenesis and axonal sprouting suggests a continuum of vascular and neural reorganization in the tissue adjacent to stroke. The changes of protein synthesis are reversible in the ischemia-resistant cortex in animals, but persist in the vulnerable regions [8]. In the vulnerable regions, apoptotic programs are induced, closely in parallel with the manifestation of delayed cell death. Thus, the recovery of protein synthesis may be a major factor influ- encing tissue survival after stroke. In addition, components of an adult neuronal growth program have been identified in the peripheral nervous system during axonal regeneration, with an overlap to axonal sprouting after stroke. The gene expression profile of the aged brain suggests an altered central nervous system environment that may exacerbate initial injury and impair neural reorganization after stroke.

In the recent past, progress in the ability to transfer proteins by protein transduction technology across the blood-brain barrier, as well as advances in neurological gene therapy, which has shown that brain defects in experimental disease models can be prevented and corrected, indicate that sufficient information is now available to contemplate radical changes in treatment strategies in patients with stroke [9]. New methods of imaging provide a powerful tool to image the penumbra and should be used to monitor the therapeutic effects.

\section{References}

1 Schaller B, Graf R, Jacobs AH: Ischaemic tolerance: a window to endogenous neuroprotection? Lancet 2003;362:1007-1008.

2 Sharp FR, Lu A, Tang Y, et al: Multiple molecular penumbras after focal cerebral ischemia. J Cereb Blood Flow Metab 2000;20: 1011-1032.

3 Roth A, Gill R, Certa U, et al: Temporal and spatial gene expression patterns after experimental stroke in a rat model and characterization of PC4 as a potential regulator of transcription. Mol Cell Neurosci 2003;22:353364.
4 Jin K, Mao XO, Eshoo MW, et al: Microarray analysis of hippocampal gene expression in global cerebral ischaemia. Ann Neurol 2001; 50:93-103

5 Vikman P, Edvinsson L: Gene expression profiling in the human middle cerebral artery after cerebral ischemia. Eur $\mathbf{J}$ Neurol, in press.

6 Ginsberg MD: Adventures in the pathophysiology of brain ischemia: penumbra, gene, expression, neuroprotection: the 2002 Thomas Willis Lecture. Stroke 2003;34:214-223.
7 Kilic E, Dietz GPH, Herrmann DM, et al: Intravenous TAT-Bcl- $\mathrm{X}_{\mathrm{L}}$ is protective when delivered before and after middle cerebral artery occlusion in mice. Ann Neurol 2002;52:617622 .

8 Hermann DM, Kilic E, Hata R, et al: Relationship between metabolic dysfunctions, gene responses and delayed cell death after mild focal cerebral ischemia in mice. Neuroscience 2001; 104:947-955

9 Schaller B, Graf R: Cerebral ischemic preconditioning. An experimental phenomenon or a clinical important entity of stroke prevention? J Neurol 2002;249:1503-1511.

\section{Erratum}

This editorial has been submitted without informed consent of the mentioned author Mathias Bähr who is not willing to co-author this paper and whose name is therefore retracted from the author list. 